\title{
Phytophthora nicotianae var parasitica pathogène du piment en Tunisie
}

\author{
MB Allagui ${ }^{1 *}$, JT Marquina ${ }^{2}$, A Mlaiki 1 \\ 1 INRAT, laboratoire de cryptogamie, rue Hedi-Karray, 2080 Ariana, Tunisie \\ 2 INSPV, Crta La Coruna Km 7.5, 28040 Madrid, Espagne
}

(Reçu le 26 janvier 1995 ; accepté le 22 juin 1995)

\begin{abstract}
Résumé - Une espèce de Phytophthora différente de Phytophthora capsici, extrêmement dommageable et fréquente sur piment en Tunisie est identifiée. Les symptômes se caractérisent par un brunissement des racines, du collet et un flétrissement rapide des plantes ou plantules atteintes. Ce pathogène sévit aussi bien sous abri-serre en hiver qu'en plein champ l'été. L'identification des souches du parasite fondée sur leurs caractéristiques morphologiques, biologiques, et l'inoculation artificielle de plants sains de piment, montrent que l'agent pathogène est Phytophthora nicotianae var parasitica sensu Stamps et al (1990), synonyme de Phytophthora parasitica sensu Tucker (1931). Une étude sur l'homothallisme, l'hétérothallisme et les 2 types sexuels de compatibilité génétique A1 et A2 chez les souches testées a été entreprise. Les variations morphologiques constatées au niveau des souches ainsi que la dénomination de cette espèce de Phytophthora sont discutées.
\end{abstract}

piment / Phytophthora nicotianae var parasitica / flétrissement / taxonomie

Summary - Phytophthora nicotianae var parasitica pathogen of the pepper in Tunisia. A Phytophthora species infecting pepper plants in Tunisia was identified. The symptom characteristics are collar and root rot leading to diseased plants or plantlets and rapid wilting. The pathogen attacks the plants in the greenhouse during winter as well as in the field during the summer. The identification of the parasite strain using morphological and biological behavior and artificial inoculation of healthy pepper plants showed that the species causing the disease is Phytophthora nicotianae var parasitica sensu Stamps et al (1990), which is synonymous with Phytophthora parasitica sensu Tucker (1931). The study of the homothallism, heterothallism and sexual genetic compatibility of the A1 and A2 types was undertaken. The morphological variations observed in the strains and the Phytophthora sp taxonomy are discussed.

pepper / Phytophthora parasitica / wilting / taxonomy

\section{INTRODUCTION}

La maladie du flétrissement du piment (Capsicum annuum $L$ ) sévit dans plusieurs régions tunisiennes (le cap Bon, le Sahel, la basse vallée de la Medjerba, la région de
Bizerte...). Elle se développe aussi bien en plein champ que sous abri-serre. Les plantes malades montrent un flétrissement général et rapide sans jaunissement. Le collet et les racines présentent un rétrécissement et un brunissement apparent bien délimité pouvant progresser vers la tige

\footnotetext{
* Correspondance et tirés à part
} 
mais sans atteindre en général la ramification primaire. Le flétrissement est dû à la perturbation de la circulation de l'eau dans les vaisseaux désorganisés sous l'effet du parasite. Aux premiers stades de linfection, de rares pourritures ont pu être observées sur les feuilles ou les fruits. Après le dépérissement, la masse foliaire et fructifère reste attachée à la plante pendant que les fruits immatures prennent une couleur rougeâtre.

Cette maladie a une incidence économique considérable. Le flétrissement se manifeste par le dessèchement des plantes jeunes ou en pleine production, ce qui représente, pour l'agriculteur, une chute de rendement importante allant jusqu'à la destruction totale au champ ou sous serre.

II n'existe pas d'étude approfondie sur les causes de cette maladie. A priori, elle pourrait être attribuée à Phytophthora capsici, à Verticillium dahliae ou même à un excès d'eau qui entraîne l'asphyxie des racines (Palazon et al, 1977 ; Palazon et Palazon, 1989). Dans son inventaire sur les maladies des cultures maraîchères en Tunisie, Davet (1967) a décrit les symptômes de cette maladie observée sur piment à Nabeul en signalant qu'un Phytophthora $\mathrm{sp}$ est incriminé. Plus tard, dans un document interne à I'INRAT, Mahjoub (1979) a cité, sans décrire la méthode d'isolement ou d'identification, Phytophthora capsici comme étant l'agent infectieux du piment en Tunisie, produisant au collet des plantes des pourritures molles descendant vers les racines. D'autre part, Moens et Ben
Aïcha (1982) ont utilisé, pour tester l'efficacité de plusieurs fongicides anti-mildiou, de la terre venant de parcelles portant des plants de piment flétris, en considérant, sans aucune justification taxonomique apparente, que le pathogène à l'origine de ce flétrissement était Phytophthora capsici. Les services de vulgarisation du ministère de l'Agriculture (1976) considèrent encore que les symptômes de brunissement racinaire et de flétrissement des plantes de piment sont produits par $P$ capsici.

Les éléments rassemblés dans notre étude montrent qu'il ne s'agit pas de $P$ capsici, parasite connu depuis longtemps sur piment (Léon, 1922), mais plutôt d'une autre espèce de Phytophthora non décrite auparavant comme pathogène sur le piment mais cependant présente à grande échelle en Tunisie. II s'agit de Phytophthora nicotianae var parasitica sensu Stamps et al (1990), synonyme de Phytophthora parasitica sensu Tucker (1931).

\section{MATÉRIEL ET MÉTHODES}

\section{Matériel cryptogamique}

Les régions prospectées pour la présence du flétrissement du piment ont été la basse vallée de la Medjerda, la région de Bizerte, le cap Bon et le Sahel. La culture traditionnelle du piment y occupe de grandes surfaces.

Au cours de cette analyse, l'étude a été faite sur 13 souches issues de différentes plantes et venant des 4 régions précitées (tableau I).

Tableau I. Caractéristiques des échantillons de piment présentant des symptômes de flétrissement au champ et révélant, après isolement sur milieu sélectif, la présence de Phytophthora nicotianae var parasitica.

$\begin{array}{ccccccc}\begin{array}{c}\text { Code } \\ \text { souches }\end{array} & \begin{array}{c}\text { Date } \\ \text { d'échantillonnage }\end{array} & \begin{array}{c}\text { Type } \\ \text { d'exploitation }\end{array} & \begin{array}{c}\text { Lieu de } \\ \text { prélèvement }\end{array} & \begin{array}{c}\text { Plante } \\ \text { hôte }\end{array} & \begin{array}{c}\text { Stade } \\ \text { végétatif }\end{array} & \begin{array}{c}\text { Taux } \\ \text { d'attaque (\%) a }\end{array} \\ 1 & 26 / 5 / 91 & \text { Primeur } & \text { Manouba } & \text { Différentes variétés } & \text { Production } & 5 \text { à } 10 \\ 2 & 8 / 10 / 91 & \text { Saison } & \text { Gornata } & \text { Meski } & \text { Fin production } & 1 \text { à } 5 \\ 3,219,220, & 3 / 3 / 92 & \text { Primeur } & \text { Bkalta } & \text { B26 et Beldi } & \begin{array}{c}\text { Plantes jeunes } \\ \text { et adultes }\end{array} & 60 \text { à } 100 \\ 221,222 & 11 / 8 / 92 & \text { Saison } & \text { Besbessia } & \text { Beldi } & \text { Production } & 20 \text { à } 30 \\ 4 & 25 / 8 / 92 & \text { Saison } & \text { Cherfech 2 } & \text { Variété locales } & \text { Production } & 10 \text { à } 20 \\ 5 & 8 / 4 / 93 & \text { Primeur } & \text { Chott Mariem } & \text { B26 } & \text { Production } & 1 \text { à } 5 \\ 6 & 1 / 7 / 93 & \text { Saison } & \text { Korba } & \text { Variétés locales } & \text { Production } & 30 \text { à } 70\end{array}$

a Estimation visuelle du nombre de plantes flétries par rapport au total des plantes de la parcelle. 


\section{Les milieux de culture}

Pour éviter le développement des champignons secondaires et des bactéries, le milieu sélectif des pythiacées mis au point par Ponchet et al (1972) a été utilisé pour tous les isolements. Ce milieu se compose de : 1 I d'eau distillée, $10 \mathrm{~g}$ de gélose, $10 \mathrm{~g}$ de maltéa moser, $250 \mathrm{mg}$ de pénicilline G (400000 UI), $250 \mathrm{mg}$ de polymixine $\mathrm{B}$ (200000 UI), $15 \mathrm{mg}$ de bénomyl et $100 \mathrm{mg}$ de pentachloronitrobenzène. La stérilisation est faite à l'autoclave à $110^{\circ} \mathrm{C}$ pendant $30 \mathrm{~min}$. Les racines et le collet de l'échantillon sont lavés, séchés puis déposés sur le milieu sélectif.

Deux autres milieux de culture ont été utilisés : l'un à base d'une décoction de petit pois ( $P G$ ) (Boccas, 1978), l'autre de pomme de terre (PDA) (Rapilly, 1968).

Après confirmation de leur appartenance au genre Phytophthora, les souches, conservées dans des tubes contenant du milieu PDA à la température du laboratoire, sont repiquées tous les 3 ou 4 mois.

\section{Identification de l'espèce}

\section{Morphologie}

Elle est basée sur des observations microscopiques de la morphologie du champignon cultivé sur les milieux $P G$ et PDA. Le milieu $P G$, riche en stérols (Boccas, 1978), est favorable à la formation des organes sexuels, ainsi que des sporocystes. Les chlamydospores sont produites plus abondamment dans l'eau stérile. Les caractères mis en évidence et représentant une valeur taxonomique importante sont comparés avec ceux retenus dans la clef de Stamps et al (1990).

\section{Biométrie des sporocystes et des oospores}

Une étude biométrique des sporocystes concernant la longueur ( $L$ ), la largeur ( $(\mathrm{l})$ et le rapport $L / l$ a été menée sur 3 souches $(1,2$ et 3 ) cultivées sur PDA pendant $10 \mathrm{j}$. Pour chacune des souches, 100 mesures ont été réalisées.

Les souches, placées au début en culture simple (sur milieu PG, à l'obscurité et à $25^{\circ} \mathrm{C}$ ) pour la production d'oospores, ont été par la suite confrontées aux 2 types complémentaires de référence $\mathrm{A} 1$ et $\mathrm{A} 2$ appartenant à $P$ capsici. Un croisement intraspécifique a été fait pour les souches dont les oospores n'ont pas été produites, ni en culture simple, ni par croisement avec $P$ capsici. Le témoin est représenté par la confrontation entre le type $\mathrm{A} 1$ de $P$ capsici et une deuxième référence appartenant à $P$ nicotianae var nicotianae de type A2 (tableau II). Le diamètre de 20 oospores par souche ayant produit les organes sexuels en culture simple ou en confrontation a été mesuré.

Tableau II. Compatibilité génétique des souches (homothallisme/hétérothallisme) de $P$ nicotianae var parasitica. Incubation sur milieu PG à l'obscurité et à $25^{\circ} \mathrm{C}$ pendant au moins $5 \mathrm{j}$.

\begin{tabular}{|c|c|c|c|c|c|c|c|c|c|c|c|}
\hline \multirow[t]{2}{*}{ Code isolats } & \multicolumn{4}{|c|}{$\begin{array}{l}\text { Oospores en } \\
\text { culture simple } \\
\text { (3 répétitions) }\end{array}$} & \multicolumn{3}{|c|}{$\begin{array}{c}\text { Oospores en } \\
\text { croisement avec } \\
\text { P capsici }\end{array}$} & \multicolumn{3}{|c|}{$\begin{array}{l}\text { Oospores en } \\
\text { croisement } \\
\text { intraspécifique }\end{array}$} & \multirow[t]{2}{*}{$\begin{array}{l}\text { Type de compatibilité } \\
\text { génétique observé }\end{array}$} \\
\hline & $R 1$ & $R 2$ & R3 & Type & $A 1$ & $A 2$ & Type & $5(A 1)$ & $3(A 2)$ & Type & \\
\hline 1 & - & - & - & $\ldots$ & $\ldots$ & $\ldots$ & $\ldots$ & $\ldots$ & $\ldots$ & $\ldots$ & $\ldots$ \\
\hline 2 & + & + & - & hom & - & + & $\mathrm{A} 1$ & $\ldots$ & $\ldots$ & $\ldots$ & hom/A1 \\
\hline 3 & - & - & - & $\ldots$ & + & - & $\mathrm{A} 2$ & + & $\ldots$ & $\mathrm{A} 2$ & $\mathrm{~A} 2$ \\
\hline 219 & - & + & $\ldots$ & hom & * & + & hom & $\ldots$ & $\ldots$ & $\ldots$ & hom \\
\hline 220 & + & + & - & hom & * & + & hom & $\ldots$ & $\ldots$ & $\ldots$ & hom \\
\hline 221 & - & - & - & $\ldots$ & - & - & $\ldots$ & + & - & $\mathrm{A} 2$ & $\mathrm{~A} 2$ \\
\hline 222 & - & - & - & $\ldots$ & - & - & $\ldots$ & + & - & $\mathrm{A} 2$ & $\mathrm{~A} 2$ \\
\hline 4 & + & + & + & hom & + & + & hom & $\ldots$ & $\ldots$ & $\ldots$ & hom \\
\hline 5 & - & - & - & $\ldots$ & - & + & $\mathrm{A} 1$ & $\ldots$ & $\ldots$ & $\ldots$ & $\mathrm{A} 1$ \\
\hline 6 & - & - & - & $\ldots$ & - & - & $\ldots$ & - & + & A1 & $\mathrm{A} 1$ \\
\hline 14 & - & - & - & $\ldots$ & - & - & $\ldots$ & * & + & hom & hom \\
\hline 18 & + & + & $\ldots$ & hom & * & + & hom & $\ldots$ & $\ldots$ & $\ldots$ & hom \\
\hline 19 & * & - & $\ldots$ & & - & + & $\mathrm{A} 1$ & * & + & hom & hom $/ A 1$ \\
\hline P capsici (A1) & - & - & - & $\ldots$ & - & + & $\mathrm{A} 1$ & $\ldots$ & $\ldots$ & $\ldots$ & A1 confirmé \\
\hline$P n$ var $n$ (A2) & - & - & - & $\ldots$ & + & - & $\mathrm{A} 2$ & $\cdots$ & $\ldots$ & $\ldots$ & A2 confirmé \\
\hline
\end{tabular}

+ : formation d'oospores sur la ligne de confrontation pour les cultures doubles et naissance d'oospores près de l'implant mycélien pour les cultures simples; ${ }^{*}$ : formation d'un petit nombre d'oospores en secteurs limités généralement à côté de l'implant mycélien, aussi bien en culture simple que double, mais absence d'oospore sur la ligne de confrontation pour les cultures doubles ; - : absence d'oospore dans tout le milieu de culture; hom : tendance vers l'homothallisme, les oospores sont formées en secteurs limités et non distribuées de manière homogène sur tout le milieu ; ... : essai n'ayant pas donné de résultat exploitable ou non effectué ; $P n$ var $n$ : Phytophthora nicotianae var nicotianae; R1, R2, R3 : répétitions 1, 2 et $3 ; \mathrm{A} 1, \mathrm{~A} 2$ : types de compatibilité génétique. 


\section{Croissance à $36^{\circ} \mathrm{C}$}

Des explants de $9 \mathrm{~mm}$ de diamètre prélevés dans chaque souche ont été déposés dans des boîtes de Petri de $90 \mathrm{~mm}$ contenant $15 \mathrm{ml}$ de milieu PDA et placé à l'obscurité à $36 \pm 0,5^{\circ} \mathrm{C}$ pendant $96 \mathrm{~h}$.

Deux mesures, en position perpendiculaire, de la croissance radiale du mycélium sur le milieu gélosé ont été effectuées après la période d'incubation (3 répétitions par souche). $P$ capsici et $P$ nicotianae var nicotianae ont été introduites dans l'essai comme témoins.

\section{Infection artificielle}

L'isolat 1 a été inoculé artificiellement sur des plantes de piment de variétés locales et étrangères au stade 912 feuilles (Allagui, 1994). Du terreau, préalablement désinfecté par autoclavage à $120^{\circ} \mathrm{C}$ pendant $30 \mathrm{~min}$, a été utilisé comme substrat. On dépose au collet de chaque plante $25 \mathrm{ml}$ d'une suspension de zoospores titrée à 10000 par $\mathrm{ml}$. Les zoospores sont obtenues en utilisant la méthode suivante : après élevage du champignon sur PDA pendant $10 \mathrm{j}$ à $25^{\circ} \mathrm{C}$, des fragments mycéliens sont immergés dans l'eau distillée en boîte de Petri pendant 3 à $7 \mathrm{j}$. De nombreuses zoospores sont libérées dans l'eau après refroidissement à $4^{\circ} \mathrm{C}$ pendant $1 \mathrm{~h}$. La température diurne des plantes inoculées était maintenue à un maximum de $25^{\circ} \mathrm{C}$ mais celle de la nuit pouvait descendre au dessous de $15^{\circ} \mathrm{C}$.

\section{RÉSULTATS}

\section{Papille des sporocystes}

Tous les sporocystes observés sont pourvus de papilles très proéminentes (fig 1). La zone transparente de la papille est étroite et les sporocystes sont généralement uni-papillés, rarement munis de 2 papilles ou plus. La seule présence de papille très apparente chez les sporocystes limite la recherche de l'espèce aux 2 groupes I et II de la clef de Stamps et al (1990).

\section{Position de l'anthéridie par rapport à l'oogone}

Les organes sexuels de ce Phytophthora sont incapables de se former sur milieu PDA et en présence de lumière du jour. Cependant, le milieu PG incubé à l'obscurité et sous une température de $25^{\circ} \mathrm{C}$ permet une formation abondante d'oospores à partir du $5^{\mathrm{e}}$ jour.

Certaines souches ont la capacité de produire des oospores (tableau II) indifféremment en cul-

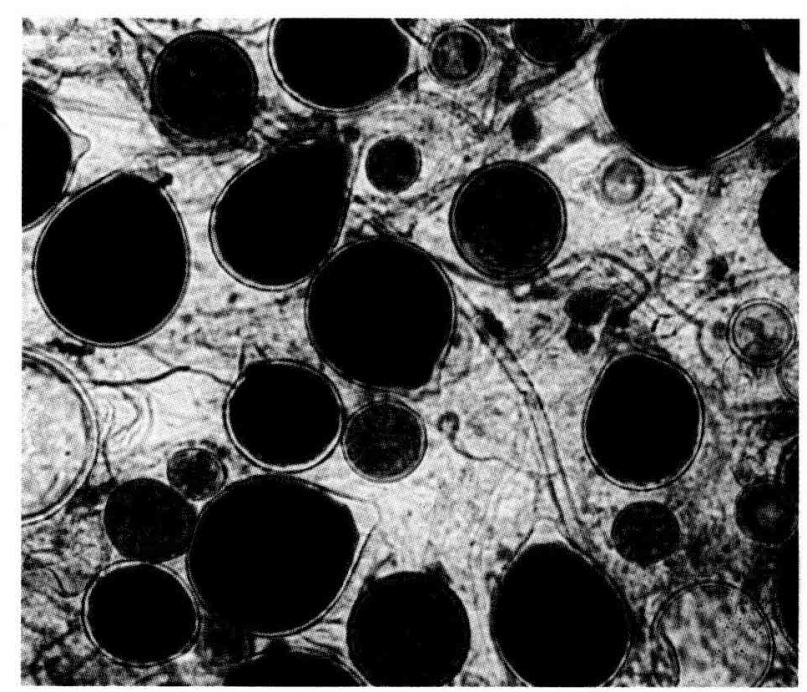

Fig 1. Sporocytes avec papilles proéminentes et sans pédicelle. Entre les sporocystes apparaissent les chlamydospores de forme sphérique.

ture simple ou en confrontation avec un type sexuel complémentaire (souches 4, 18, 219, 220) : ce sont des souches homothalliques. En revanche, d'autres ne produisent des oospores que si elles sont opposées à un type conjugal complémentaire (souches $3,5,6,14,221,222$ ). Les croisements inter- et intraspécifiques révèlent l'existence de 2 types sexuels $A 1$ et $A 2$ pour les souches étudiées.

D'autre part, les croisements intraspécifiques (entre nos souches isolées du piment) sont plus favorables à une production abondante d'oospores que les confrontations interspécifiques (entre nos souches et la référence $P$ capsicl). Ceci apparaît chez les couples complémentaires formés entre la souche 5 de type $A 1$ ou 3 de type A2 et les souches $6,14,221$ et 222 dont le type conjugal n'a pas été déterminé en croisement interspécifique par manque de formation d'oospores (tableau II).

La position amphigyne de l'anthéridie par rapport à l'oogone est la règle pour toutes les souches (fig 2), ce qui permet de rattacher l'espèce recherchée au groupe II puisque le groupe I réunit les espèces à position paragyne.

\section{Chlamydospores}

Sur milieu PG, on peut observer facilement des chlamydospores. Elles sont généralement sphériques et terminales. Dans l'eau, leur nombre est encore plus élevé, avec une position intercalaire ou terminale. La production de chlamydospores 


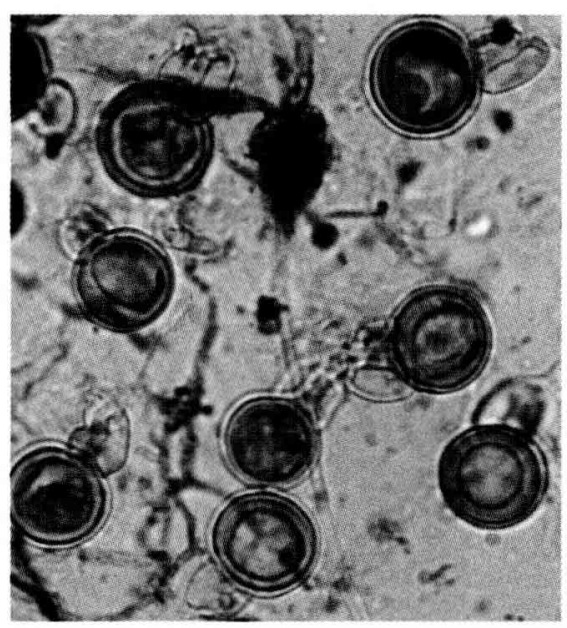

Fig 2. Oospores plérotiques et anthéridies en position amphigyne par rapport à l'oogone.

par toutes les souches (fig 1), aussi bien en milieu gélosé que dans l'eau, les apparentent aux 2 espèces suivantes : $P$ palmivora MF1 et MF2 et $P$ nicotianae. Cependant, $P$ capsici se distingue par l'absence de chlamydospores.

\section{Pédicelle et forme des sporocystes}

En général, les sporocystes des souches se caractérisent par une forme plus ou moins sphérique à ovoïde avec un pédicelle très court, à peine visible au microscope (fig 1). Le pédicelle des sporocystes est considéré comme étant un caractère de diagnostic stable (Al-Hedaithy et
Tsao, 1979a, b, cité par Erwin, 1983). La taille très courte du pédicelle des sporocystes permet d'exclure catégoriquement l'appartenance de nos souches à l'espèce $P$ capsici. La forme sphérique à ovoïde des sporocystes est plutôt une caractéristique de $P$ nicotianae que de $P$ palmivora dont les sporocystes sont en majorité ellipsoïdes.

\section{Croissance à $36^{\circ} \mathrm{C}$}

Toutes les souches analysées montrent une croissance mycélienne à $36^{\circ} \mathrm{C}$ (fig 3 ). À cette température élevée, la croissance mycélienne moyenne a variée de $5,3 \mathrm{~mm}$ à $9,5 \mathrm{~mm}$ en $4 \mathrm{j}$ à l'obscurité.

Dans le groupe II de la clef de Stamps et al (1990), la croissance mycélienne à une température supérieure à $35^{\circ} \mathrm{C}$ représente un critère taxonomique important pour $P$ nicotianae. Étant donné que $P$ palmivora n'est pas capable de croître à $36^{\circ} \mathrm{C}$, nous déduisons que nos souches se rattachent à l'espèce $P$ nicotianae (var nicotianae ou var parasitica).

\section{Biométrie des sporocystes et des oospores}

\section{Dimensions des sporocystes}

La variation intra-souches de la longueur et de la largeur des sporocystes est importante, variant respectivement entre $33-75$ et $23-56 \mu \mathrm{m}$ (tableau III). La variation inter-souches de ces 2

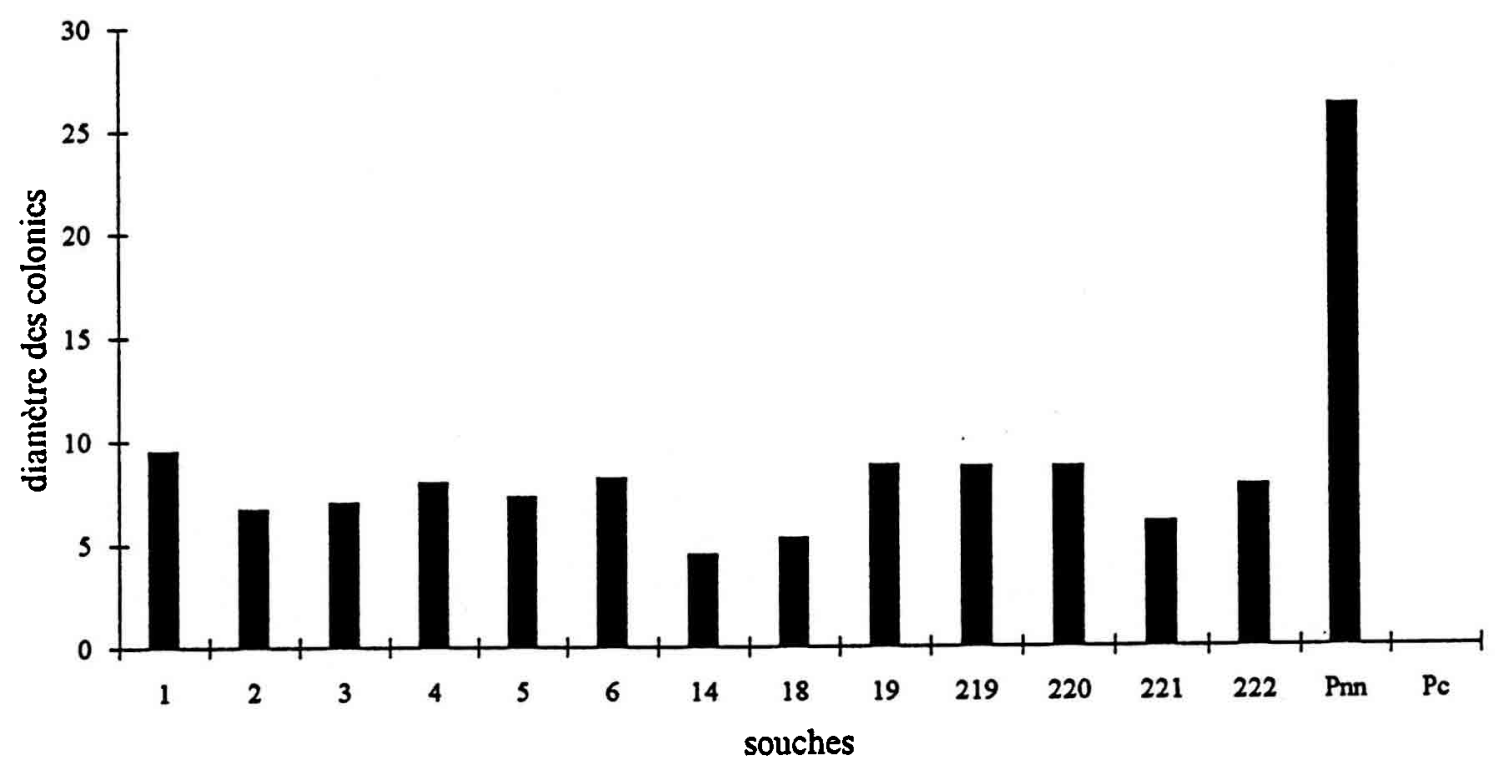

Fig 3. Croissance radiale moyenne $(\mathrm{mm})$ du mycélium de souches de $P$ nicotianae var parasitica incubées à $36^{\circ} \mathrm{C}$ et à l'obscurité pendant 96 h sur PDA ( $\mathrm{Pnn}=P$ nicotianae var nicotianae, $\mathrm{PC}=P$ capsici). 
caractères est plus réduite que la précédente mais la différence entre souches demeure significative. Cependant, le rapport $L /$, malgré sa grande variation intra-souches, ne met pas en évidence de différence significative entre les souches.

La moyenne de la longueur, de la largeur et de leur rapport établie sur 300 sporocystes a été respectivement de $53,4 \mu \mathrm{m}, 38,7 \mu \mathrm{m}$ et 1,38 .

\section{Diamètre des oospores}

Les oospores sont toujours plérotiques (fig 2). Les oospores formés en culture simple ou en croisement sont de taille relativement petite. Leur diamètre oscille entre 14 et $26 \mu \mathrm{m}$. La moyenne par souche varie de 18,8 à $23,5 \mu \mathrm{m}$ et la moyenne générale effectuée sur 100 oospores est de $21,6 \mu \mathrm{m}$ (valeur moyenne d'une importance particulière pour la taxonomie de Phytophthora nicotianae var parasitica). Néanmoins, dans les mêmes conditions, les oospores formées par le couple témoin $P$ nicotianae var nicotianae de type $\mathrm{A} 2$ et $P$ capsici de type $\mathrm{A} 1$ ont un diamètre moyen de $28,2 \mu \mathrm{m}$ qui est significativement plus élevé que le diamètre des oospores de nos souches.

\section{Autres caractères distinctifs}

Les observations microscopiques ont révélé que les hyphes, dont les parois sont irrégulières, ont un aspect coréallö̈de (fig 4). Cette caractéristique, attribuée à $P$ nicotianae var parasitica, est commune à toutes les souches observées. En revanche, $P$ nicotianae var nicotianae est décrite

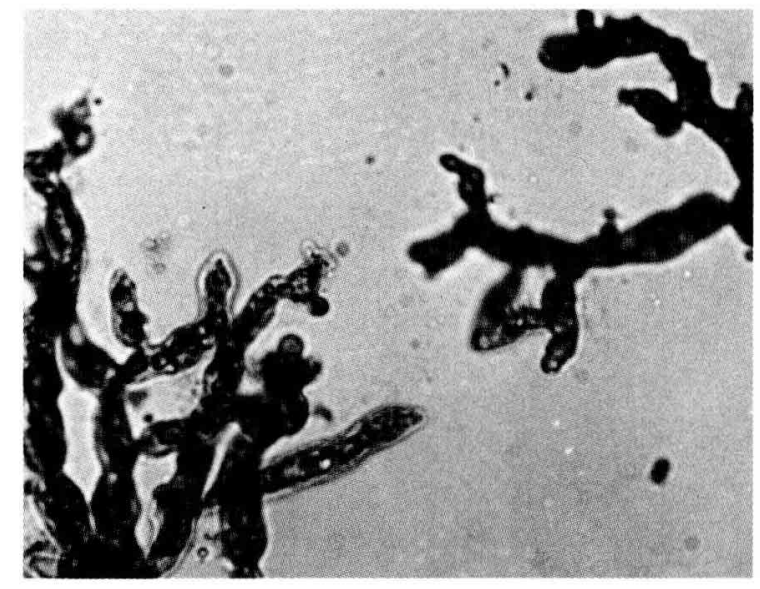

Fig 4. Mycélium corallö̈de et à parois irrégulières.

comme ayant des hyphes dont les parois sont uniformes.

D'autre part, les renflements mycéliens, normalement présents chez $P$ nicotianae var nicotianae, n'ont été mis en évidence chez aucune de nos souches cultivées sur milieu PG et PDA.

\section{Test de pouvoir pathogène}

Les inoculations artificielles des plants de piment de quelques variétés locales et étrangères ont entraîné des mortalités à partir du $17 \mathrm{e}$ jour après l'inoculation. Les symptômes identiques à ceux des plantes infectées naturellement (figs 5 et 6 ) et le réisolement du même champignon utilisé en inoculation permet de satisfaire au postulat de Koch, indiquant que $P$ nicotianae var parasitica

Tableau III. Longueur $(\mu \mathrm{m})$, largeur $(\mu \mathrm{m})$, rapport (longueur/largeur) et limite de variation de ces 3 caractères chez les sporocystes de 3 souches de Phytophthora nicotianae var parasitica isolées du piment et mises en culture sur PDA).

\section{Souches}

Longueur $(L)$ *

et limite de variation
Largeur $(I)$ *

et limite de variation
Rapport (LII)*

et limite de variation

$\begin{array}{lll}51,9^{a} & 37,2^{a} & 1,39 \text { a } \\ (33-66) & (23-47) & (1,1-1,9) \\ 54,1^{b} & 39,6^{b} & 1,37 \text { a } \\ (37-66) & (23-56) & (1,1-1,6) \\ & & \\ 54,2^{b} & 39,3 \mathrm{~b} & 1,38 \mathrm{a} \\ (37-75) & (28-52) & (1,2-2,4)\end{array}$

* Valeurs moyennes de 100 sporocystes. Les moyennes d'une même colonne sont significativement différentes selon le test de Duncan au seuil de $5 \%$ lorsqu'elles ont un indice différent. 


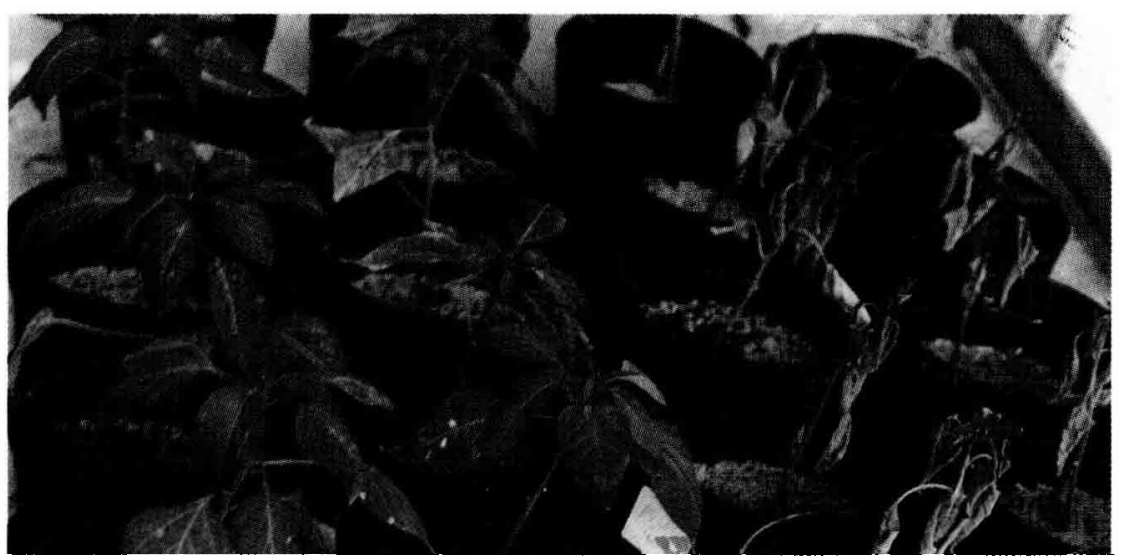

Fig 5. Aspect (à droite) de plants de piment après inoculation artificielle par $P$ nicotianae var parasitica, par rapport au témoin non inoculé (à gauche), indemne.

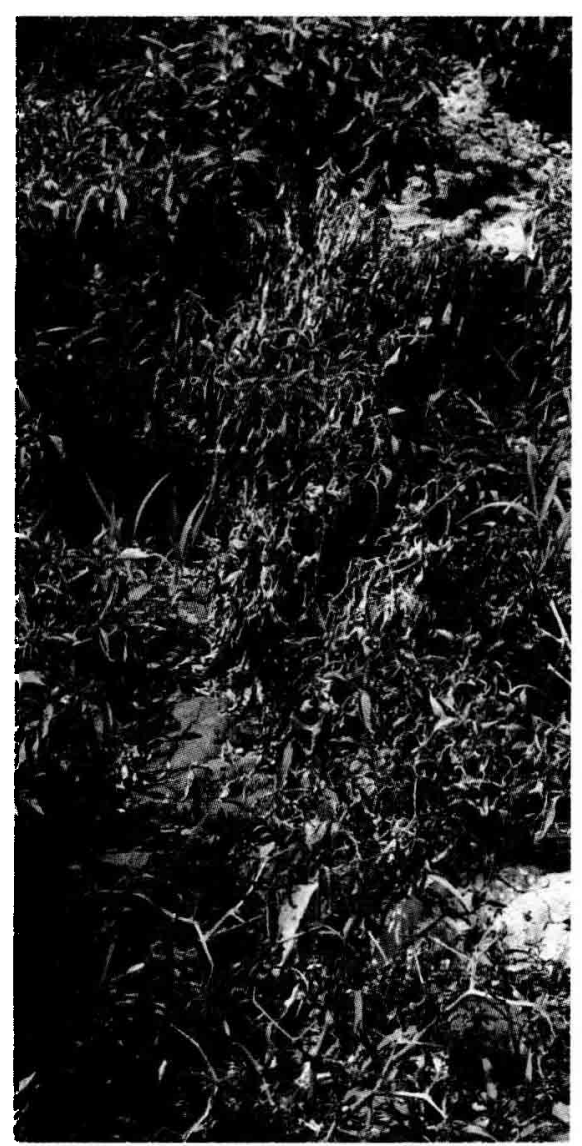

Fig 6. Plant de piment naturellement atteint de flétrissement et révélant, après isolement, la présence de $P$ nicotianae var parasitica.

est l'agent causal de la maladie du flétrissement du piment.

\section{DISCUSSION ET CONCLUSION}

Nous avons montré que le pathogène qui produit des pourritures, des brunissements des racines, du collet, un flétrissement général et rapide des plantes de piment en Tunisie n'est pas le $P$ cap- sici, parasite connu depuis longtemps. Les résultats de cette analyse prouvent nettement, malgré les imperfections des clefs de détermination relative à Phytophthora, que l'agent infectieux de cette maladie est le Phytophthora nicotianae var parasitica sensu Stamps et al (1990). Cette espèce est la seule de son genre identifiée comme parasite du piment en Tunisie.

Il est admis que $P$ capsici est une espèce de Phytophthora capable d'attaquer le piment. Aucun auteur, semble-t-il, n'a démontré qu'en plus de $P$ capsici, le piment peut être affecté par une autre espèce de Phytophthora. Léon (1922) et Tucker (1931) ont indiqué que $P$ capsici est la seule espèce du genre capable d'infester le piment. Cette opinion a été ensuite admise par Satour et Butler (1968) pour développer une étude approfondie de l'hérédité de la pathogénicité de $P$ capsici sur le piment et la tomate.

Quant à $P$ parasitica, il est traditionnellement considéré à un parasite des citrus (Klotz et al, 1958 ; Tsao, 1969), de la tomate (Messiaen et al, 1991) et de l'œillet (Hine et Aragaki, 1952 ; Tramier et Andréoli, 1969 ; Tello, 1990) mais non du piment (Palazon, 1988). Très récemment, Bartual et al (1991) ont isolé à partir de plants de piment victimes de flétrissement, outre $P$ capsici prédominant en Espagne, $P$ parasitica, dans une zone continentale réduite à Ciudad Real et Toledo. Notre étude révèle que $P$ nicotianae var parasitica est responsable des pourritures racinaires et du flétrissement du piment en Tunisie. Plus de 300 autres souches que nous avons isolées et identifiées postérieurement montrent les mêmes caractéristiques que les souches figurant dans cette étude.

Du point de vue taxonomique, les caractères morphologiques et biologiques étudiés coïncident, selon la clef de Stamps et al (1990), avec Phytophthora nicotianae var parasitica. Cette dénomination n'est pas acceptée par tous les 
auteurs et suscite certaines controverses. Tucker (1931) a suggéré de donner le nom Phytophthora parasitica à toutes les souches de Phytophthora qui se distinguent par le type de croissance en milieu de culture, la papille des sporocystes, l'abondance de chlamydospores en milieu de culture, la position amphigyne de l'anthéridie et la capacité de croître à $35^{\circ} \mathrm{C}$ sur milieu maïs gélosé. Ce même auteur propose que toutes les souches de $P$ parasitica qui attaquent les plantes jeunes ou adultes de tabac Nicotiana tabacum soient dénommées $P$ parasitica var nicotianae, nomenclature admise par Gooding et Lucas (1959), Savage et al (1968) et Tsao (1969). Cependant, cette classification n'a pas été retenue par Waterhouse (1963) qui a appelé cette espèce $P$ nicotianae en la subdivisant en 2 variétés : var nicotianae et var parasitica. Plus tard ce thème a continué d'intéresser les chercheurs. Erwin (1983) a remarqué qu'en utilisant les critères morphologiques de Waterhouse (1963) toutes les souches de tabac $P$ parasitica var nicotianae sensu Tucker (1931) ne sont pas nécessairement synonymes de $P$ nicotianae sensu Waterhouse (1963). Cet auteur pense qu'il est évident de nommer la forme spéciale tabac $P$ parasitica fsp nicotianae. Waterhouse et al (1983) suggèrent que la clef qu'ils ont établie en 1978, malgré sa simplicité, ne résoud pas la complexité existante dans la dénomination de certaines espèces comme nicotianae-parasitica et le groupe de Palmivora. À propos de l'espèce de Phytophthora que nous avons isolée du piment en Tunisie, nous considérons comme plus logique de l'appeler Phytophthora parasitica sensu Tucker (1931).

La longueur ( $L$ ), la largeur (I) et le rapport $L / I$ des sporocystes sont des critères parmi d'autres, utilisés pour la séparation des espèces de Phytophthora (Waterhouse, 1963). En nous basant sur l'analyse de 3 de nos isolats, nous avons montré les grandes variations intrasouches et inter-souches de $L$ et $I$, c'est pourquoi nous n'avons pas ataché à ces caractères une importance taxonomique majeure. Le rapport $L / l$, bien que la variation intra-souches soit importante, ne montre pas de différence significative inter-souches. À cause de la grande variabilité des mesures de la longueur, de la largeur et de leur rapport chez les sporocystes de $P$ parasitica, Tucker (1931) a décidé de ne pas accorder une valeur taxonomique à ces caractères. Toutefois, nos résultats montrent que le rapport L/l demeure utile, compte tenu des moyennes non significatives entre souches et la moyenne générale 1,38 inférieure à la limite supérieure 1,40 fixée par Tucker (1931) et Waterhouse (1963) pour $P$ parasitica. Pour trouver un rapport caractéristique de l'espèce, il nous semble intéressant de considérer la moyenne d'un grand nombre de sporocystes pris au hasard et appartenant à plusieurs souches au lieu de se limiter à quelques sporocystes d'une seule souche.

La variation entre souches n'est pas restreinte à la morphologie des sporocytes puisqu'elle s'affirme également dans la croissance mycélienne à $36^{\circ} \mathrm{C}$, par le diamètre des oospores et plus particulièrement au niveau de l'homothallisme et I'hétérothallisme, quoique Stamps et al (1990) dans leur clef n'accordent pas une valeur taxonomique à ce phénomène. Toutefois, Sansome (1980, cité par Waterhouse et al, 1983), Tello (1990) et Bartual et al (1991) indiquent l'existence de souches homothalliques et d'autres hétérothalliques dans $P$ nicotianae var parasitica. En revanche, $P$ capsici est bien considéré encore comme une espèce réellement hétérothallique, ce qui est bien un autre facteur de distinction entre les 2 espèces.

Dès lors que nous avons révélé l'existence d'une espèce de Phytophthora parasitant le piment en Tunisie, différente de ce qui est connu par ailleurs, il convient de poursuivre son étude, en particulier en ce qui concerne sa pathogénicité, son épidémiologie et les méthodes de lutte.

\section{REMERCIEMENTS}

Ce programme de recherche est soutenu par la Fondation internationale pour la science (FIS), Suède. Les auteurs remercient sincèrement $R$ Perrin et P Camporota (INRA, Dijon) pour leur participation à la correction de cet article.

\section{RÉFÉRENCES}

Allagui MB (1994) Phytophthora nicotianae var parasitica resistance ability of some pepper varieties. Capsicum Eggplant News 13, 93-96

Al-Hedaithy SSA, Tsao PH (1979a) The effects of culture media and sporulation methods on caducity and pedicel length of sporangia in selected species of Phytophthora. Mycologia 71, 392-401

Al-Hedaithy SSA, Tsao PH (1979b) Sporangium pedicel length in Phytophthora species and the consideration of its uniformity in determining sporangium caducity. Trans Br Mycol Soc 72, 1-13

Bartual R, Marsal JI, Carbonell A, Tello JC, Campos T (1991) Genetica de la resistancia a Phytophthora capsici Léon en pimiento. Bol San Veg Plagas 17, 1-124 
Boccas B (1978) La reproduction sexuelle chez les Phytophthora: ses voies et quelques-unes de ses conséquences génétiques. Thèse doc es sci nat, univ Paris-Sud, Orsay

Davet P (1967) Les maladies des solanées maraîchères en Tunisie. Ann Inst Natl Rech Agr Tunisie 40, 1-43

Erwin DC (1983) Variability within and among species of Phytophthora. In : Phytophthora, its Biology, Taxonomy, Ecology and Pathology (DC Erwin, S Bartniki-Garcia S, PH Tsao, eds), Ann Phytopathol Soc, St Paul, MN, États-Unis

Gooding GV, Lucas GB (1959) Factors influencing sporangial formation and zoospore activity in Phytophthora parasitica var nicotianae. Phytopathology 49, 277-281

Hine RB, Aragaki H (1952) A new wilt disease of carnations in Hawaii caused by Phytophthora parasitica. Hawaii Fin Sci 11, 6

Klotz LJ, DeWolfe TA, Wong PP (1958) Decay of fibrous root of citrus. Phytopathology 48, 616-622

Léon LH (1922) Stem and fruit blight of peppers caused by Phytophthora capsici sp Nov. Phytopathology 12, 401-408

Mahjoub M (1979) Principales maladies cryptogamiques et bactériennes sur plantes cultivées en Tunisie. Document interne à I'INRAT, 378

Moens M, Ben Aïcha B (1982) Possibilités de lutte préventive et curative contre le mildiou du piment Phytophthora capsici (Léon). Med Fac Landbouww Rijksuniv Gent 47, 953-959

Messiaen CE, Blancard D, Rouxel F, Lafon R (1991) Les maladies des plantes maraîchères ( $3^{e}$ éd). INRA, Paris

Palazon C, Gil R, Palazon I (1977) Contribucion al estudio de la fermedad conocida como «tristeza»o "seca» del pimiento. Publ CRIDA 03, Zaragoza, $200 p$

Palazon C (1988) Estudios de los posibles metodos de control de la "tristeza" o "seca" del pimiento. Tesis Doctoral, Univers Politec Valencia, $231 \mathrm{p}$

Palazon C, Palazon I (1989) Estudios epidemiologicos sobre la "tristeza" del pimiento en la zona del Valle Medio del Ebro. Bol San Veg Plagas 15, 233262
Ponchet J, Ricci P, Andréolli C, Augé G (1972) Méthodes sélectives d'isolement du Phytophthora nicotianae fsp parasitica (Dastur) Waterh à partir du sol. Ann Phytopathol 42, 97-108

Rapilly F (1968) Les techniques de mycologie en pathologie végétale. Ann Épiphyt 19, ( $n^{\circ} \mathrm{HS}$ )

Sansome E (1980) Reciprocal translocation heterozygosity in heterothallic species of Phytophthora and its significance. Trans Br Mycol Soc 74, 175-185

Savage EJ, Clayton CW, Hunter JH, Brenneman JA, Laviola C, Gallegly ME (1968) Homothallism, heterothallism and interspecific hybridization in the genus Phytophthora. Phytopathology 58, 1004-1021

Satour MM, Butler EE (1968) Comparative morphological and physiological studies on the progenies from intraspeicifc matings on Phytophthora capsici. Phytopathology 58, 183-192

Service de vulgarisation du ministère de l'Agriculture en Tunisie (1976) La défense des cultures en Afrique du Nord (Office allemand de la coopération technique, ed), fasc «solanées", $69 \mathrm{p}$

Stamps DJ, Waterhouse FJ, Newhook FJ, Hall GS (1990) Revised tabular key to the species of Phytophthora. Mycol Papers 162

Tello JC (1990) La podridumbre del cuello y de la base del tallo del clavel causada por Phytophthora de Bary en los cultivos espanoles. Cuadernos de Fitopalogia/1er trimestre 9-12

Tramier R, Andréoli C (1969) Biologie et écologie du Phytophthora nicotianae f parasitica (Dastur) Wateh parasite du collet de l'œillet. Ann Phytopathol (HS), 171-176

Tsao PH (1969) Studies on the saprophytic behavior of Phytophthora parasitica in soil. In : Proc First Intern Citrus Symp (HD Chapman, ed), 3, 1221-1230

Tucker CM (1931) Taxonomy of the genus Phytophthora de Bary. Res Bull Mo Agric Exp St, 153 p

Waterhouse GM (1963) Key to the species of Phytophthora de Bary. Mycol Papers 92, 1-22

Waterhouse GM, Newhook FJ, Stamps DJ (1983) Present criteria for classification of Phytophthora. In : Phytophthora, its Biology, Taxonomy, Ecology and Pathology (DG Erwin, S Bartniki-Garcia, PH Tsao, eds), Ann Phytopathol Soc, Saint Paul, MN, États-Unis 